\title{
Cirugía coronaria exclusiva con dos mamarias en "Y": resultados a corto plazo
}

\author{
Enrique Seguel ${ }^{1,2}$, Aleck Stockins ${ }^{1,2}$, Luis Figueroa², Roberto González ${ }^{1,2}$, Emilio Alarcón ${ }^{1,2}$, Camila Quiñones $^{3}$. \\ (1) Departamento de Cirugía, Facultad de Medicina, Universidad de Concepción; \\ (2) Centro Cardiovascular, Hospital Guillermo Grant Benavente de Concepción \\ (3) Alumno de Medicina, Universidad de Concepción.
}

Introducción: La cirugía de revascularización miocárdica (CRM) exclusiva con arterias mamarias internas en Y (BIMA-Y) podría ofrecer los mejores injertos en términos de durabilidad y menores tasas de eventos adversos en el largo plazo.

Objetivo: Analizar los resultados a corto plazo de esta técnica quirúrgica en un hospital universitario chileno.

Métodos: Estudio prospectivo de los pacientes intervenidos de CRM con técnica de BIMA-Y entre diciembre 2010 a mayo 2014. Se incluyeron 67 pacientes, 59 hombres, con edad media 58,6 $\pm 8,5$ años. Cinco (5) tenían enfermedad de dos vasos y 62 de 3 vasos. El EuroSCORE aditivo fue $2.3 \mathrm{y}$ el logístico $2,18 \%$. Todas las cirugías se realizaron por esternotomía media con circulación extracorpórea. Ambas arterias mamarias internas se disecaron usando técnica esqueletizada. La arteria mamaria interna derecha (RIMA) se seccionó y anastomosó con la arteria mamaria interna izquierda
(LIMA). La pared anterior se revascularizó con LIMA y las paredes lateral e inferior con RIMA. El seguimiento se extendió hasta Junio del 2015.

Resultados: Se efectuaron 3,8 anastomosis/paciente: 109 LIMA (67 a arteria descendente anterior, 37 a una arteria diagonal y 5 para otros objetivos) y 145 RIMA (64 a una primera arteria marginal, 23 a una segunda marginal y 58 a descendente posterior). La revascularización fue completa en todos los casos Hubo 1 infarto perioperatorio, 6 fibrilaciones auriculares, 1 neumonía, 1 dehiscencia esternal y 5 infecciones superficiales. No hubo mortalidad operatoria ni reoperaciones por sangrado. El seguimiento medio fue $39,3 \pm 12,1$ meses. Cuatro pacientes fallecieron y hubo 2 reintervenciones.

Conclusiones: La técnica BIMA-Y permitió una revascularización completa con una baja tasa de complicaciones y efectos adversos en el corto plazo. 


\section{Myocardial revascularization using both mammary arteries "in Y": short term results}

Background: Myocardial revascularization with both mammary arteries in "Y" (Y-BIMA) might lead to longer graft duration and better clinical results.

Aim: To analyze short term results of Y-BIMA in a series of patients operated on at a Chilean university hospital.

Methods: Patients operated on using Y-BIMA between December 2010 and May 2014 were analyzed. There were 67 patients (59 males) aged $58.6 \pm 2.3$ years old. Five patients had 2 vessel disease and 62 patients 3 vessel disease. The additive Euroscore was $2.3 \%$ and the logistic score was $2.18 \%$. All operations were performed through a medial sternotomy using extracorporeal circulation. Both mammary arteries were dissected and skeletonized. The RIMA was divided and anastomosed to the LIMA. The anterior myocardial was revascularized through the LIMA while the lateral and inferior walls were revascularized through the RIMA. Results: An average 3.8 anastomosis per patient were performed: 67 LIMA to the LAD, 37 to the diagonal artery, and 5 to other targets (total 109 LIMA); RIMA was used in 145 arteries: 64 to a first marginal, 23 to a second marginal and 58 to a posterior descending artery. Revascularization was complete in all cases. One patient had a postoperative myocardial infarction, 6 developed atrial fibrillation, 1 a pneumonia, 1 a sternal dehiscence and 5 had superficial infections. There was no surgical mortality nor reoperations for bleeding. Patients were followed an average of $39.2 \pm 12.1$ months. Four patients died and 2 were reoperated on during follow up.

Key Words: coronary surgery, coronary artery bypass grafting, double mammary, BIMA, short terms results. 
Introducción: Desde fines de los años 70, la cirugía coronaria ha demostrado tener un importante rol en el tratamiento de la enfermedad coronaria permitiendo una mejoría en los síntomas, de la capacidad funcional y de la sobrevida. En grupos de pacientes seleccionados los resultados pueden superar a los del tratamiento médico ${ }^{1-3}$. A pesar de los buenos resultados a corto y mediano plazo mostrados en estos grupos, el seguimiento en el largo plazo mostró una pérdida del beneficio de la cirugía respecto al tratamiento médico, con una tendencia de las curvas de sobrevida a igualarse en ambos grupos después de los 11 años. ${ }^{4-5}$

$\mathrm{Al}$ analizar la serie total de los 1240 pacientes incluidos en estos estudios podemos observar que la arteria mamaria interna (AMI) se utilizó sólo en un 9,9\% de las cirugías ${ }^{5}$. A principios de los años 80 el grupo de cirujanos de la Cleveland Clinic, demostró que la permeabilidad de los puentes venosos era inferior a la de la mamaria, y que el uso de esta última se asociaba a una menor tasa de eventos (infartos, hospitalizaciones y necesidad de revascularización repetida) y a una mejor sobrevida de los pacientes ${ }^{6-7}$. Actualmente, el uso de mamaria izquierda (LIMA) como injerto sobre la arteria descendente anterior es considerado como el "gold standard" en revascularización miocárdica. ${ }^{8}$ Estudios posteriores nacionales y extranjeros demostraron que el uso de dos arterias mamarias se asociaba a una mejor sobrevida comparado con el uso de una sola, incluso en pacientes añosos. Este beneficio se observa después de 10 años de seguimiento ${ }^{9-13}$.

Un inconveniente del uso de dos mamarias es que la mamaria derecha pediculada sólo puede ser utilizada en ramas proximales de la arteria circunfleja (pasando por el seno transverso) o el tronco de la coronaria derecha. En 1991, J.A. Barra publicó una serie de 25 pacientes intervenidos utilizando ambas arterias mamarias en forma exclusiva. Para ello propuso seccionar la mamaria derecha y anastomosarla a la mamaria izquierda en "Y" permitiendo, con el uso de anastomosis secuenciales, revascularizar todos los territorios del corazón ${ }^{14-15}$. Estudios posteriores del mismo grupo mostraron buenos resultados clínicos y angiográficos a un año de seguimiento ${ }^{16}$. El 2007, el equipo de la Clínica Marie Lannelongue, en París, publicó los resultados del control angiográfico utilizando dos mamarias en configuración en "Y" en pacientes con enfermedad de tres vasos para una revascularización completa del corazón. El control angiográfico precoz demostró una excelente permeabilidad, tanto de la "Y" como de las anastomosis distales. ${ }^{17}$

A fines de 2010 iniciamos el uso de esta técnica en nuestro centro y el objetivo de esta comunicación es mostrar los resultados inmediatos y del seguimiento a corto plazo (3 años) en los primeros 67 pacientes.

\section{Métodos}

La realización de este estudio fue aprobada por el Comité Ético Científico del Servicio de Salud Concepción.

\section{Pacientes:}

La selección de pacientes se realizó considerando la anatomía coronaria (lesiones proximales $>75 \%$, vasos coronarios de calibre $>1,5 \mathrm{~mm}$, no calcificados, no intramiocárdicos).

La serie está compuesta por 67 pacientes intervenidos entre el $1^{\circ}$ de diciembre de 2010 y el 30 de mayo de 2014 en el Hospital Guillermo Grant Benavente de Concepción, lo que corresponde al 5,6\% de las cirugías coronarias totales realizadas en ese período $(\mathrm{N}=1191)$.

Hubo una marcada preponderancia de hombres y la edad promedio era la habitual en estos pacientes. Los factores de riesgo cardiovascular fueron los habituales para la hipertensión arterial y dislipidemia y algo menos de la mitad de los pacientes eran fumadores. De especial interés para el análisis de este trabajo es la alta prevalencia de Diabetes $(40.3 \%)$.

Clínicamente, más de $4 / 5$ de los pacientes presentaba angina crónica estable, y algo menos de 1/5 angina inestable Un tercio de los pacientes había experimentado un infarto de menos de 90 días de evolución. Aproximadamente $9 \%$ de los pacientes tenía angioplastía coronaria previa.

La gran mayoría de los pacientes presentaba lesión de 3 vasos y solo $7.5 \%$ de 2 vasos. La fracción de eyección del ventrículo izquierdo era normal en la mayoría de los pacientes. El riesgo operatorio fue relativamente bajo: por EuroSCORE aditivo (18) fue 2,3 $\pm 1,7$ (rango 0 - 10 puntos) y por EuroSCORE logístico (19) fue de 2,18 $\pm 2,2 \%$ (rango $0.88-16,8 \%$ ), (Tabla 1 ).

\section{Técnica quirúrgica}

Todas las intervenciones se realizaron con anestesia general por esternotomía media. Ambas arterias mamarias internas se disecaron con técnica esqueletizada y sin abrir las pleuras (Figura 1). Una vez completada la disección de ambos injertos se procedió a la heparinización plena de los pacientes, a la confección de las jaretas y a la canulación arterial en la aorta ascendente y venosa atrio - cava. Sin entrar en circulación extracorpórea, se exploró el corazón e identificaron los objetivos a revascularizar, se seccionó la mamaria izquierda a distal y la mamaria derecha a 
Tabla 1. Características de los pacientes

\begin{tabular}{|c|c|c|}
\hline PACIENTES & 67 & \\
\hline Hombres & 59 & $88 \%$ \\
\hline Mujeres & 8 & $12 \%$ \\
\hline Edad & $58 \pm 8,5$ años & Rango $33-81$ años \\
\hline \multicolumn{3}{|l|}{ FACTORES DE RIESGO } \\
\hline Hipetensión arterial & 49 & $73,1 \%$ \\
\hline Diabetes mellitus & 27 & $40,3 \%$ \\
\hline Tabaquismo & 27 & $40,3 \%$ \\
\hline Dislipidemia & 20 & $29,8 \%$ \\
\hline \multicolumn{3}{|l|}{ HISTORIA CARDIOVASCULAR } \\
\hline Angina crónica & 56 & $83,6 \%$ \\
\hline Angina inestable & 11 & $16,7 \%$ \\
\hline Infarto Reciente (<90 días) & 23 & $34,3 \%$ \\
\hline Angioplastía previa & 6 & $8,9 \%$ \\
\hline \multicolumn{3}{|l|}{ LESIONES CORONARIAS } \\
\hline Lesión de dos vasos & 5 & $7,5 \%$ \\
\hline Lesión de tres vasos & 62 & $92,5 \%$ \\
\hline Fracción de Eyección del VI & $58 \pm 9,9 \%$ & Rango $35-75 \%$ \\
\hline \multicolumn{3}{|l|}{ RIESGO OPERATORIO } \\
\hline EuroSCORE aditivo & $2,3 \pm 1,7$ & Rango 0 - 10 puntos \\
\hline EusoSCORE logístico & $2,18 \pm 2,2 \%$ & Rango $0,88-16,8 \%$ \\
\hline
\end{tabular}

proximal y distal, y se construyó una anastomosis entre ambas de forma término - lateral en "Y" con sutura de polipropileno 8-0 (Prolene ${ }^{\circledR}$, Ethicon, Johnson \& Johnson, Brazil) (Figura 2). Terminada la anastomosis se comprobó su permeabilidad observando el flujo hacia ambas ramas. Finalizada la construcción de la "Y", se inició la CEC, pinzó la aorta y detuvo el corazón mediante una solución de cardioplejia.

La revascularización de la cara anterior se realizó utilizando la mamaria izquierda, mediante la confección de anastomosis latero - laterales en las ramas diagonales y término - lateral en la descendente anterior. Posteriormente se luxó el corazón y procedió a revascularizar la cara lateral con la mamaria derecha mediante la confección de anastomosis latero - laterales en las ramas marginales y la cara inferior mediante la confección de anastomosis término - lateral sobre la arteria descendente posterior (Figura 3). Terminadas todas las anastomosis distales, se revisó la hemostasia, se devolvió el corazón a su lugar y despinzó la aorta. Una vez que el corazón hubo retomado su actividad se procedió a la salida de CEC, se revirtió la heparina con protamina y cerró la toracotomía de manera habitual. El detalle de la técnica quirúrgica utilizada puede encontrarse en la publicación previa de nuestro grupo ${ }^{20}$.

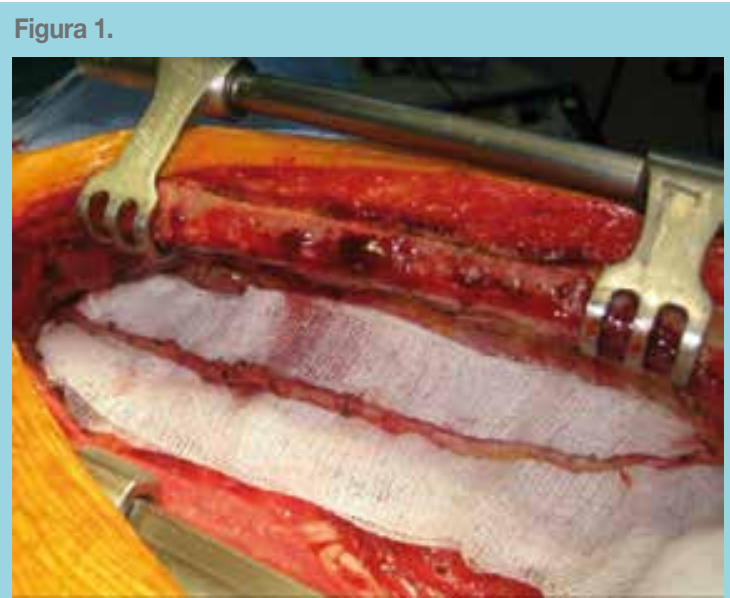

Figura 1: Arteria mamaria interna izquierda disecada con técnica esqueletizada.

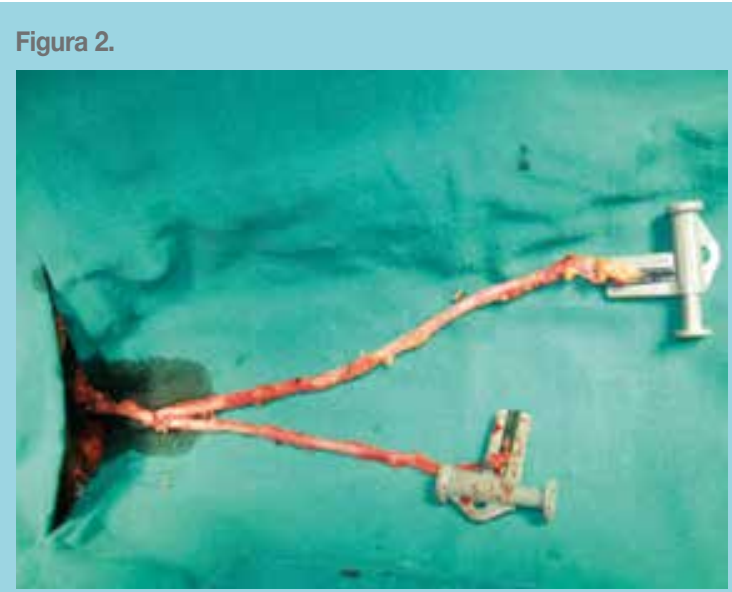

Figura 2: Anastomosis en " $Y$ " terminada entre las arterias mamarias internas derecha e izquierda.

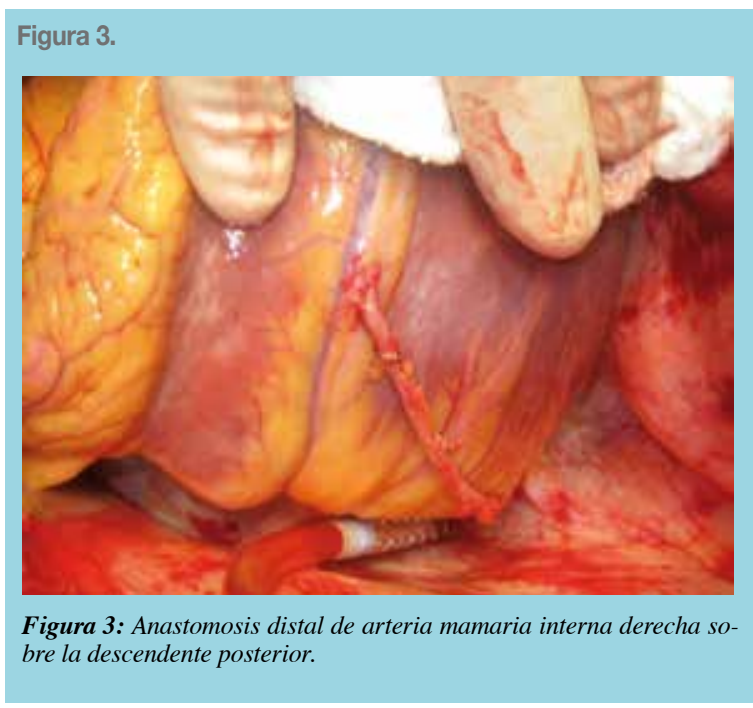


Se tabuló el número y objetivos de las anastomosis distales, duración (en minutos) de la intervención, horas de ventilación mecánica post operatoria, débito de los drenes durante las primeras 24 horas y necesidad de transfusión de hemoderivados.

Las complicaciones se definieron como: quirúrgicas (hemorragia, re operación), neurológicas (accidente vascular encefálico, déficit transitorio, coma), renales (insuficiencia renal aguda con o sin necesidad de diálisis), cardíacas (arritmias, infarto peri operatorio, insuficiencia cardíaca), infecciones (herida operatoria, mediastinitis, extremidad inferior, infección urinaria), pulmonares (ventilación mecánica $>48$ horas, neumonía), vasculares (disección aórtica, isquemia extremidad) y otras (digestivas). La mortalidad operatoria se consideró hasta los 30 días post cirugía o hasta el alta hospitalaria si el paciente permaneció más de un mes hospitalizado.

No se realizó coronariografía de control y sólo se estudiaron aquellos que tuvieron indicación por clínica (síntomas, eventos agudos). El seguimiento se completó hasta el 30 de junio de 2015. Los eventos clínicos y re intervenciones fueron obtenidos de los registros de las fichas clínicas de los pacientes, la base de datos del hospital y los registros del laboratorio de hemodinamia. La sobrevida se obtuvo de acuerdo a los datos del Servicio de Registro Civil e Identificación. Se consignan la mortalidad alejada y los eventos cardiovasculares mayores: infarto (IAM), accidente cerebro vascular (AVE) y reintervención.

\section{Resultados}

\section{Cirugías}

Se realizaron 254 anastomosis distales, (promedio 3,8 \pm 0.63 puentes/paciente). Corresponden a 109 anastomosis con mamaria izquierda (67 a la descendente anterior; 37 a una diagonal y 5 a otro objetivo) y a 145 anastomosis con mamaria derecha (64 a una $1^{\circ}$ marginal; 23 a una $2^{\circ}$ marginal y 58 a una inter ventricular posterior).

La duración promedio de la cirugía fue de $174 \pm 40 \mathrm{~min}$ (2,9 horas). Se extubaron en pabellón casi todos los pacientes. El sangrado post operatorio y necesidad de transfusiones fueron bajos (Tabla 1). No hubo re - intervenciones por sangrado.

\section{Complicaciones}

Se presentaron complicaciones médicas en 8 pacientes: $1(1,5 \%)$ IAM peri operatorio, $6(8,9 \%)$ arritmias (AC x FA) y $1(1,5 \%)$ neumonía. No se presentaron complicaciones neurológicas, renales ni ventilación mecánica prolongada. Hubo complicaciones quirúrgicas en 6 pacientes: 1
(1,5\%) dehiscencia esternal sin infección (re intervención para re - alambraje esternal), $5(7,4 \%)$ infecciones superficiales de herida operatoria. No hubo casos de mediastinitis. No hubo mortalidad operatoria (Tabla 2).

\section{Tabla 2: Resultados inmediatos}

\section{CIRUGÍAS}

Anastomosis distales

$3,8 \pm 0,63$ puentes/paciente

Duración de la cirugía

$174 \pm 40$ minutos

Extubación en pabellón

63 pacientes

$94 \%$

Débito drenes 24 horas

$691 \pm 300 \mathrm{cc}$

Transfusiones 11 pacientes

$16,4 \%$

\section{COMPLICACIONES MÉDICAS}

Infarto peri operatorio

Arritmias

Neumonía

6

Neurológicas

Renales

$0 \%$

(

Ventilación prolongada

$0 \%$

COMPLICACIONES QUIRÚRGICAS

Infección herida operatoria

Dehiscencia esternal

Re operaciones por sangrado

Mediastinitis

FALLECIDOS

\section{Seguimiento}

Se logró el seguimiento del $100 \%$ de los pacientes. El seguimiento promedio fue 39,3 312,1 meses (rango: 12 - 54 meses). Hubo 4 fallecidos (1.8/100 pacientes /año). Fallecieron a los 11, 15, 22 y 42 meses de la cirugía: 3 pacientes fallecieron de causas cardiovasculares (paro cardio - respiratorio en uno e insuficiencia cardíaca descompensada en dos) y uno por sepsis. La sobrevida global de la serie fue de $94 \%$.

Durante el seguimiento ningún paciente presentó un infarto agudo al miocardio (IAM), ni accidente vascular encefálico (AVE). Dos pacientes requirieron de una re intervención por síntomas: Un paciente fue estudiado con coronariografía a los 7 meses y se encontraron todos los puentes permeables. Otro paciente se estudió a los 20 meses, encontrando una oclusión de la AMI izquierda luego de la anastomosis en "Y". Se realizó una angioplastía e implante de stent a la descendente anterior. La tasa combinada de eventos fue de 2,9/100 pacientes /año. 


\section{Discusión}

Los resultados presentados muestran una baja morbilidad y ausencia de mortalidad en la revascularización miocárdica utilizando la tecina de BIMA_Y.

A pesar de la evidencia, el uso de doble mamaria en cirugía coronaria es poco frecuente. En Estados Unidos y Europa corresponde a menos del 5\% del total de cirugías coronarias $^{21-23}$. Durante el período de estudio utilizamos doble mamaria sólo en $112(9,4 \%)$ de los 1191 pacientes intervenidos en nuestro centro. Sin embargo, durante estos años hemos aumentado progresivamente el uso de dos mamarias en revascularización miocárdica, pasando de un 5,4\% el año 2012 a 7,9\% el año 2013 hasta un 16\% el año 2014. Entre las razones para explicar el poco uso de dos mamarias destacan una mayor duración de la cirugía (debido al tiempo de disección de la segunda mamaria) y una mayor tasa de infecciones o problemas de cicatrización del esternón, especialmente en los pacientes añosos, obesos y diabéticos.

El único estudio randomizado que comprara el uso de una versus dos mamarias es el ART trial, actualmente en desarrollo, y confirmó una mayor tasa de complicaciones de la esternotomía en los pacientes operados con dos mamarias comparada con aquellos en que se utilizó una sola $(1,9 \%$ vs $0,6 \%$ ). Sin embargo estas complicaciones no afectaron la mortalidad a 30 días (1,2\% en ambos grupos) ni a un año (2,5\% vs $2,3 \%$ respectivamente) ${ }^{24}$

Una alternativa técnica de la disección mamaria, propuesta por Keeley en 1987, consiste en la esqueletización de la arteria. La arteria es disecada sin las venas ni el tejido circundante y tiene una mayor longitud y flujo que las arterias disecadas de forma tradicional ${ }^{25}$. Estudios posteriores mostraron que el uso de la AMI esqueletizada se asocia a menor sangrado post operatorio, mejor función pulmonar y a una menor tasa de infecciones que la disección con todo el pedículo ${ }^{26-29}$. Esta disminución en la incidencia de complicaciones esternales se observa incluso en diabéticos ${ }^{30-34}$. En esta serie se utilizó la técnica esqueletizada en la disección mamaria. La tasa de complicaciones esternales fue baja $(1,5 \%)$ y consistió en una dehiscencia esternal sin infección. Otros 5 pacientes presentaron infecciones superficiales de herida operatoria que fueron manejadas con antibióticos y curaciones ambulatorias, y no requirieron de una re intervención ni la prolongación de su estadía hospitalaria.

La técnica propuesta por Barra permite aumentar al doble la longitud de la AMI derecha, alcanzar las arterias de la pared lateral e inferior del corazón y lograr una revascularización completa del corazón con estos injertos. Debido a que la anastomosis entre ambas mamarias puede comprometer la revascularización de todo el corazón y a la necesidad de realizar anastomosis secuenciales, es una técnica más demandante que la cirugía tradicional y no ha sido adoptada universalmente.

En la selección de pacientes privilegiamos a pacientes más jóvenes y de bajo riesgo operatorio. Si bien la serie incluye a 6 pacientes mayores de 70 años, la mayoría (52\%) eran menores de 60 años. El EuroSCORE aditivo mostró un bajo riesgo en $38(56,7 \%)$, riesgo moderado en $24(38,8 \%)$ y riesgo alto en $8(11,9 \%)$, cifras menores que las observadas en pacientes operados con técnicas convencionales Así mismo, se eligieron pacientes con lesiones coronarias mayores a $75 \%$ para evitar el fenómeno de competencia de flujo. Este problema puede afectar a los injertos arteriales, los que tienden a atrofiarse cuando son anastomosados a arterias con lesiones menos significativas y en los que el flujo por la arteria nativa es mayor que el flujo por el injerto. $35-36$

No hubo mortalidad operatoria en esta serie y la tasa de complicaciones fue baja, lo cual puede deberse a que se trataba de un grupo seleccionado de pacientes de bajo riesgo. Los resultados a tres años muestran una baja mortalidad e incidencia de eventos cardiovasculares mayores. Sólo dos pacientes de la serie han sido re - estudiados, encontrando todos los puentes permeables en uno y una oclusión de la AMI izquierda posterior a la anastomosis con la AMI derecha, lo que probablemente se debe a un problema técnico. Estos resultados son comparables a otras series publicadas con esta técnica. ${ }^{17-37}$

\section{Conclusión}

La cirugía coronaria exclusiva con dos arterias mamarias en "Y" permitió la revascularización de todos los territorios del corazón con una baja tasa de complicaciones y de eventos en el corto plazo. El seguimiento a largo plazo de series mayores de pacientes permitirá determinar si esta técnica se traduce en mejores resultados clínicos que la cirugía tradicional. 


\section{Referencias:}

1.- MURPHY M, HULTGREN HN, DETRE K, THOMSEN J, TAKARO T. Treatment of Chronic Stable Angina. A preliminary report of survival data of the randomized veterans administration cooperative study. N Eng J Med 1977; 297 : 621 - 627.

2.- VARNAUSKAS E, OLSSON SB, CARLSTRÖM E, KARLSSON T. and the European Coronary Surgery Group. Long Terms Results of Prospective Randomised Study of Coronary Artery Bypass Surgery in Stable Angina Pectoris. The Lancet 1982: 2: $1173-1182$.

3.- DAVIS KB, GILLESPIE MJ, KENNEDY, JW KRONMAL RA AND THE CASS INVESTIGATORS. Coronary Artery Surgery study (CASS): a randomized trial of coronary artery bypass surgery. Circulation 1983; 68: 939 - 950.

4.- VARNAUSKAS E. Twelve Year Follow up Of Survival In The Randomized European Coronary Surgery Group. N Eng J Med 1988; 319: 332 - 337.

5.- YUSUF S, ZUCKER D, PEDUZZI P, FISHER LD, TAKARO T, KENNEDY JW, et al. Effect Of Coronary Artery Bypass Surgery On Survival: Overwiew Of 10 - Years Results From Randomised Trials By The Coronary Artery Bypass Graft Surgery Trialists Collaboration. Lancet 1994; 344: 563 - 570.

6.- LYTLE B, LOOP F, COSGROVE D, RATLIFF N, EASLEY K, TAYLOR P. Long Term (5 To 12 Years) Serial Studies Of Internal Mammary Artery And Saphenous Vein Coronary Bypass Grafts. J Thorac Cardiovasc Surg 1985; 89: 248 - 58.

7.- LOOP F, LYTLE B, COSGROVE D, STEWART R, GOORMASTIC M, WILLIAMS G, et al. Influence Of The Internal Mammary Artery Graft On 10 Years Survival And Other Cardiac Events. N Eng J Med 1986; 314: 1 - 6.

8.- CAMERON A, DAVIS C, GREEN G, SCHAFF H. Coronary Bypass Surgery With Internal Thoracic Artery Grafts, Effects On Survival Over A 15 Year Period. N Eng J Med 1996; 334: $216-219$.

9.- FIORE A, NAUNHEIM K, DEAN P, KAISER G, PENNINGTON G, WILLMAN V, et al. Results of Internal Thoracic Ar- tery Grafting Over 15 Years: Single versus Double Grafts. Ann Thorac Surg 1990; 49: 202 - 209.

10.- LYTLE B, BLACKSTONE E, LOOP F, HOUGHTALING P, ARNOLD J, AKHRASS R, et al. Two Internal Thoracic Artery Grafts Are Better Than One. J Thorac Cardiovasc Surg 1999; 117: $855-872$.

11.- MORAN S, IRARRAZAVAL MJ, ZALAQUETT R, VILLAVICENCIO M, GARAYAR B, MUÑOZ C, et al. Revascularización Miocardica Con Arteria Mamaria Interna Bilateral. Rev Chil Cardiol 1996; 15: 4 - 8.

12.- MORAN S, IRARRAZAVAL MJ, ZALAQUETT R, VILLAVICENCIO M, GARAYAR B, MUÑOZ C, et al. Revascularizacion Miocardica Con Una Y Dos Arterias Mamarias: Resultados Clinicos Y Seguimiento Alejado. Rev Med Chile 1997; 125: $391-401$

13.- LYTLE B, BLACKSTONE E, SABIK J, HOUGHTALING P, LOOP F, COSGROVE D. The Effect of Bilateral Internal Thoracic Artery Grafting on Survival During 20 Postoperative Years. Ann Thorac Surg; 2004; 78: 2005 - 2014.

14.- BARRA JA, MONDINE P, BEZON E, MAHLAB A, RUKBI I, BRAESCO J. Revascularisation des artéres coronaires. Réimplantation en Y de l'artére mammaire interne droite darts l'artére mammaire interne gauche. Presse Med 1991; 20: 423 - 5.

15.- BARRA JA, MONDINE P, MAHLAB A, BEZON E, RUKBI I, SLIMANE AK, et al. Right internal mammary artery reimplantation into the left internal mammary artery: $\mathrm{Y}$ anastomosis. 25 cases. Ann Chir 1991; 45: 661 -666.

16.- BARRA JA, BEZON E, MANSOURATI J, RUKBI I, MONDINE P, YOUSSEF Y. Reimplantation of the right internal thoracic artery as a free graft into the left in situ internal thoracic artery (y procedure) One-year angiographic Results. J Thorac Cardiovasc Surg 1995; 109: 1042 - 8.

17.- AZMOUN A, RAMADAN R, AL-ATTAR N, KORTAS CH, GHOSTINE S, CAUSSIN C, et al. Exclusive Internal Thoracic Artery Grafting in Triple-Vessel-Disease Patients: Angiographic Control. Ann Thorac Surg 2007; 83: 2098 -102. 
18.- NASHEF SA, ROQUES F, MICHEL P, GAUDUCHEAU E, LEMESHOW S, SALAMON R. European System For Cardiac Operative Risk Evaluation (Euroscore). Eur J Cardiothorac Surg 1999; 16: 9 -13.

19.- ROQUES F, MICHEL P, GOLDSTONE AR, NASHEF SA. The Logistic Euroscore. Eur Heart J 2003; 24: 882-3.

20.- SEGUEL E, GONZÁLEZ R, STOCKINS A, ALARCÓN E, CÁRDENAS P. Técnicas quirúrgicas. Revascularización miocárdica completa con dos mamarias. Revista Chilena de Cirugía 2012; 64: 210 - 214 .

21.- PUSKAS JD. "Why did you not use both internal thoracic arteries?”. Circulation 2012; 126: 2915 - 17.

22.- TABATA M, GRAB JD, KHALPEY Z, EDWARDS FH, O'BRIEN SM, COHN LH, et al. Prevalence and variability of internal mammary artery graft use in contemporary multivessel coronary artery bypass graft surgery: analysis of the Society of Thoracic Surgeons National Cardiac Database. Circulation 2009; 120: 935 - 40.

23.- EL BARDISSI AW, ARANKI SF, SHENG S, O'BRIEN SM, GREENBERG CC, GAMMIE JS. Trends in isolated coronary artery bypass grafting: an anlysis of the Society of Thoracic Surgeons adult cardiac surgery database. J Thorac Cardiovasc Surg 2012; 143: $273-81$.

24.- TAGGART D, ALTMAN D, GRAY A, LEES B, NUGARA F, YU LM, and the ART Investigators Randomized trial to compare bilateral vs. single internal mammary coronary artery bypass grafting: 1-year results of the Arterial Revascularisation Trial (ART). Eur Heart J 2010; 31: 2470- 81.

25.- KEELEY S. The skeletonized internal mammary artery. Ann Thorac Surg 1987; 44: 324 - 325.

26.- WIMMER-GREINECKER G, YOSSEEF-HAKIMI M, RINNE T, BUHL R, MATHEIS G, MARTENS S, et al. Effect of internal thoracic artery preparation on blood loss, lung function, and pain. Ann Thorac Surg 1999; 67: 1078 - 82.

27.- MATSUMOTO M, KONISHI Y, MIWA S, MINAKATA K. Effect of different methods of internal thoracic artery harvest on pulmonary function. Ann Thorac Surg 1997; 63: 653 - 5 .
28.- BONACCHI M, PRIFTI E, GIUNTI G, SALICAA, FRATI G, SANI G. Respitarory dysfunction after coronary artery bypass grafting employing bilateral internal mammary arteries: the influence of intact pleura. Eur J Cardiothorac Surg 2001; 19: 827-833.

29.- NOERA G, PENSA P, GUELFI P, BIAGI B, LODI R, CARBONE C. Extrapleural takedown of the internal mammary artery as a pedicle. Ann Thorac Surg 1991; 52: 1292 - 1294.

30.- PARISH M, ASAI T, GROSSI E, ESPOSITO R, GALLOWAY A, COLVIN S, et al. The effects of diferent techniques of internal mammary artery harvesting on sternal blood flow. J Thorac Cardiovasc Surg 1992; 104: 1303 - 7.

31.- LYTLE B. Skeletonized internal thoracic artery grafts and wound complications. J Thorac Cardiovasc Surg 2001; 121: $695-6$.

32.- HE G, RYAN W, ACUFF T, BOWMAN R, DOUTHIT M, YANG $\mathrm{CH}$, et al. Risk factors for operative mortality and sternal wound infection in bilateral internal mammary artery grafting. J Thorac Cardiovasc Surg 1994; 107: 196 - 202.

33.- RIDDERSTOLPE L, GILL H, AHLFELDT H, RUTBERG H. Superficial and deep sternal wound complications: incidence, risk factors and mortality. Eur J Cardiothorac Surg 2001; 20: $1168-1175$.

34.- MATSA M, PAZ Y, GUREVITCH J, SHAPIRA I, KRAMER A, PEVNY D, et al. Bilateral skeletonized internal thoracic artery grafts in patients with diabetes mellitus. J Thorac Cardiovasc Surg 2001; 121: $668-74$.

35.- MORAN SV, BAEZA R, GUARDA E, ZALAQUETT R, IRARRAZAVAL MJ, MARCHANT E, et al. Predictors of radial artery patency for coronary bypass operations. Ann Thorac Surg. 200; $72: 1552-6$.

36.- TATOULIS J. Total arterial coronary revascularization-patient selection, stenoses, conduits, targets. Ann Cardiothorac Surg. 2013; 2: 499 - 506.

37.- GLINEUR D, HANET C, PONCELET A, D'HOORE W, JC FUNKEN, J RUBAY, et al. Comparison of Bilateral Internal Thoracic Artery Revascularization Using In Situ or Y Graft Configurations A Prospective Randomized Clinical, Functional, and Angiographic Midterm Evaluation. Circulation. 2008; 118: S216-S221. 